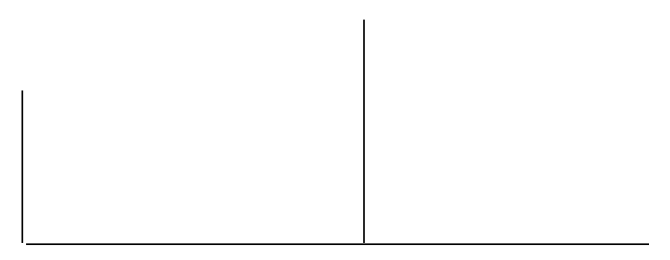

Rev. Latinoam. Psicopat. Fund., IX, 1, 44-63

\title{
Pesquisa e ensino em psicopatologia: confusões conceituais e ambigüidades nos conceitos de incoerência e desagregação no pensamento
}

\author{
Cláudio M. S. Osório \\ José Roberto Goldim \\ Richelle Becker Albrecht \\ Alexandre Maffei Machado \\ Cláudio Laks Eizirik
}

\begin{abstract}
Na investigação sobre tolerância e reações à ambigüidade e à incerteza na medicina, foram identificadas ambigüidades e confusões conceituais, que dificultam o processo ensino-aprendizagem e a pesquisa. São exemplos os conceitos confusos elou problemas na tradução de termos em psicopatologia. Os sintomas desagregação e incoerência no curso do pensamento vêm sendo definidos $e$ conceituados praticamente como sinônimos, a partir de uma distorção no entendimento de "Inkohärenz" a expressão original de Bleuler. Os avanços na pesquisa em psicopatologia, no ensino e na prática clínica dependem de clareza conceitual e precisão na nomenclatura da psicopatologia descritiva. Revisam-se os conceitos e definições de alterações do curso ou forma do pensamento em todas as edições de Kaplan \& Sadock, dada a sua larga utilização no ensino, bem como em outros textos didáticos. Cotejam-se os pontos de vista de Andreasen e Weinberger neste tema de semiologia psiquiátrica.
\end{abstract}

Palavras-chave: Psicopatologia, pesquisa (curso e forma do pensamento), incoerência, desagregação 
Descrição, caracterização e precisão na nomenclatura de sinais e sintomas, usando terminologia não-ambígua, são requisitos tanto para a prática clínica quanto para a pesquisa (Fonagy, 2001). Ambigüidades já foram consideradas impedimentos para o avanço do conhecimento (Reid, 1785). Podem ser entendidas no lato senso ou no estrito senso. No primeiro caso estão os erros e as confusões conceituais. No segundo caso, os estímulos ou informações capazes de serem interpretadas de, pelo menos, duas maneiras (vaso e/ou perfis de Rubin). Por vezes, a ambigüidade está mais no observador do que no observado, aí se incluindo as assim chamadas "ilusões de ótica" (desenhos de Escher). De forma similar, escolas ou modelos teóricos na psicanálise e na psiquiatria também podem determinar diferentes interpretações pelo observador do mesmo estímulo ou informação.

Revisando a literatura sobre a tolerância de estudantes de medicina e médicos à incerteza e à ambigüidade, particularmente no campo da tomada de decisões diagnósticas e terapêuticas, Osório, Eizirik e Goldim (2004) entenderam que as ambigüidades podem ser intrínsecas, naturais e estimulantes da curiosidade científica, e extrínsecas, construídas, artificialmente introduzidas, desestimulantes.

A revisão da tradução das obras de Freud encoraja os psiquiatras a fazerem o mesmo no seu próprio campo de conhecimento, onde se encontram alguns conceitos ambíguos, no sentido de imprecisos, confusos ou, até mesmo, errados. Convém distinguir confusão conceitual (duas informações divergentes convergindo para uma mesma interpretação) de ambigüidade (onde uma informação permite duas interpretações divergentes). Deve-se também considerar que há 


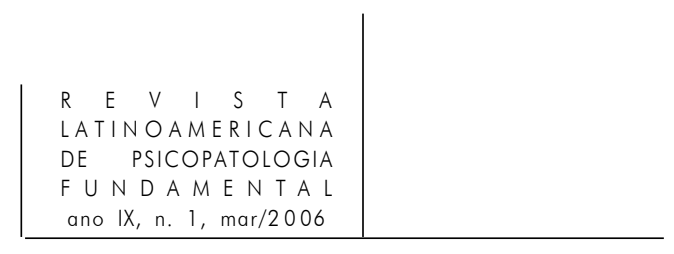

ambigüidades quase inevitáveis na psiquiatria, como a relação cérebro-mente (McHugh e Slavney, 1989) e que mesmo no campo da física existe falta de clareza conceitual. A falta de clareza na física quântica suscitou a bem-humorada observação de Niels Bohr (1996): “... clareza e verdade não são necessariamente sinônimos, podendo ser considerados conceitos complementares...” (p. 140). Embora os físicos costumem evitar as discussões conceituais (Lang da Silveira, 2003), no caso da psiquiatria e da psicanálise, esta atitude pode agravar o problema das ambigüidades. Elas podem, por exemplo, ser responsáveis por uma parte da antipatia dos estudantes de medicina pelas disciplinas de psiquiatria, em especial as de psicologia médica (Osório; Eizirik e Goldim, 2004). Além das ambigüidades, a psiquiatria convive também com as incertezas inerentes ao conhecimento científico e prática da medicina. No estudo das reações à incerteza e à ambigüidade em estudantes de medicina e médicos, destacam-se os trabalhos de Schor, Pilpel e Benbassat (2000, p. 272-80) e de Gerrity e cols. (1990, p. 724-36).

\section{Incoerência e desagregação}

Entre as diferentes situações de ambigüidade podem ser incluídas as geradas por traduções erradas, que mudam a aparência e a essência do texto original (Osório; Eizirik e Goldim, 2004). O reconhecimento dos problemas da tradução foi consagrado no aforismo traduttore, traditore. Três exemplos históricos: a idéia de um Galileu empirista e experimentador teria tido origem em um falseamento da tradução dos Discorsi, pela leitura empirista anglo-saxônica (Koyré, 1973). Outro erro foi entender, em Tomás de Aquino, a expressão Inexistenz, in-esse, que significa estar no interior de, foi traduzida como inexistente, não existente, caindo em um contra-senso ou ambigüidade, como aponta Dupuy (1996, p. 126-7). O terceiro exemplo está em Kant, onde a expressão transcendência pode ser considerada ambígua. Não é - como se costuma pensar - "o que está além da experiência". Significa "o que antecede a experiência”, de acordo com o sentido original do autor (Kant, 1989, p. 36-57).

Entre os conceitos ambíguos da psiquiatria estão os de afetividade e humor, considerados equivalentes ou sinônimos (Manley, 2000, p. 652-65; Osório, 2000; Osório, Eizirik; Goldim e Albrecht, 2003), entre outras razões por uma má tradução da palavra affect, que, dependendo do contexto, pode ser traduzida para afeto (como na rigidez afetiva) ou como humor (como no humor deprimido). Examinaremos o caso da desagregação e da incoerência no curso do pensamento, distinção relevante na discriminação diagnóstica entre as psicoses esquizofrênicas 


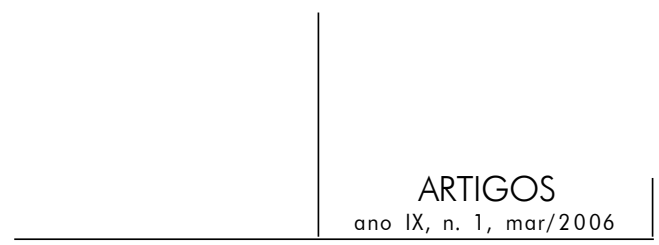

e os transtornos bipolares psicóticos. Em praticamente todas as edições do Kaplan e Sadock há confusão conceitual entre estes dois sintomas (Freedman; Kaplan e Sadock, 1967, p. 549-50; Kaplan e Sadock, 2000, p. 687). Além disso, derailment, descarrilamento, nem sempre, como deveria ser, é considerado sinônimo de loosening of associations, afrouxamento das associações ou desagregação, as expressões correspondentes ao conceito clássico de Bleuler (1911, p. 16; 1950, p. 21; 1960, p. 21-47).

Em um dos textos de semiologia mais utilizados pelos alunos de medicina, pensamento incoerente "não se distingue do pensamento esquizofrênico desagregado. A associação de idéias desprovida de lógica é característica de ambos” (Alves Brasil, M. A. e Alves Brasil, H. 1990, p. 1089-90; Porto, 1990, p. 1089-90). Bates (1987, p. 90; 1983), ao conceituar “indefinição das associações” (loosening of associations), mantém, igualmente, os conceitos confusos: “... fala na qual uma pessoa desvia-se de um assunto para outros sem qualquer relação (...) observada na esquizofrenia, em episódios maníacos e outros distúrbios psiquiátricos”. A autora conceitua incoerência como “a fala essencialmente incompreensível em virtude de conexões ilógicas e sem qualquer significado, e de mudanças bruscas de assunto (...), observada em pessoas psicoticamente afetadas (habitualmente esquizofrênicas) e também em pessoas com afasia" [grifos nossos]. O seu conceito de fuga de idéias, onde "uma pessoa muda bruscamente de um assunto para outro", permite também confundi-lo com o de desagregação.

O texto de Bleuler, de 1911, em alemão, recebeu uma versão, em inglês, em 1950. A versão em espanhol desta edição inglesa é de 1960 (Bleuler, 1950, p. 21; 1960, p. 21-47). O distúrbio da associação das idéias, dentro dos sintomas fundamentais, era assim caracterizado: “... as associações perdem a sua continuidade... [e] tendem a efetuar-se seguindo novas linhas (p. 21)... Freqüentemente o paciente abandona um pensamento de uma maneira absolutamente natural e segue com outro, muito diferente, que não tem nenhuma vinculação associativa reconhecível com o anterior... [com] saltos repentinos” (p. 27). Grifamos saltos para lembrar o termo knight's move, expressão utilizada pelos ingleses para se referir ao que denominamos desagregação, pela semelhança desta alteração de curso do pensamento com o movimento daquela peça no jogo de xadrez (Medical Research Council, 1983). Mais adiante, segue Bleuler: “... na linguagem [falada] e escrita habituais, esta peculiar desconexão dos fios associativos está combinada, freqüentemente, com outras perturbações” [do pensamento e da linguagem]. De fato, na clínica psiquiátrica, o que se escuta é um discurso que inclui desagregação do pensamento, idéias delirantes e neologismos. Por isso concordamos com Bleuler quando ele acrescenta: “... de modo que é difícil encontrar um caso puro [de desagregação do pensamento]. Nos 


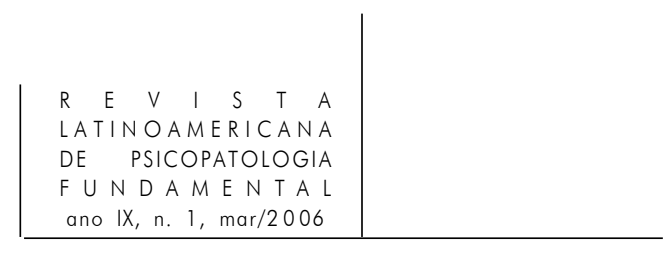

estados [esquizofrênicos] agudos, esta anomalia pode chegar a tal extremo que só excepcionalmente uma seqüência de pensamentos pode ser seguida ao longo dos seus muitos elos [unidades]. Isto foi chamado de "pensamento dissociado" [por Ziehen, em seu trabalho de 1892] $e$ "incoerência" (p. 28). ${ }^{1}$ Na versão espanhola, de 1960, "incoherencia" está também entre aspas, como "incoherence" da edição em inglês de 1950. Para Rónai (1975), as aspas servem para indicar apropriação indébita ou falsa qualidade (p. 62). Poder-se-ia, portanto, entender que o tradutor do alemão para o inglês, não encontrando palavra melhor para a expressão original de Bleuler, "Inkohärenz", traduziu-a para "incoherence". Entretanto, na língua inglesa, o uso desta quotation marks for emphasis, entre aspas, dá margem a ambigüidades, pois serve tanto para enfatizar como para indicar o contrário, isto é, que a palavra ou expressão não deveria ser tomada literalmente (Lord, 2003). Na língua alemã, menos sujeita a ambigüidades, as aspas também indicam ênfase. Em português usam-se negrito, itálico, sublinhado ou maiúsculas para destaques, e aspas para citações transcritas, além dos usos indicados por Rónai.

Impossível saber por que Bleuler escolheu "Inkohärenz", palavra de origem latina e destacando-a com as aspas para caracterizar a alteração, fundamental para ele, do curso do pensamento. Para ênfase ou para alertar o leitor que não a tomasse no sentido literal ou usual da palavra? A língua alemã dispõe de outras palavras para nomear o sintoma da esquizofrenia em exame. Enquanto desconhecíamos a expressão original, por não se ter encontrado o texto alemão no Brasil, pensouse em Sprunghäftigkeit e Hanglosigkeit. ${ }^{2}$ A primeira delas expressaria razoavelmente bem o conceito de pensamento desagregado, um salto, como no "knight's move" do xadrez. A segunda sugeriria o curso escorregadio, frouxo ou, melhor ainda, "sem gancho", sem referência, de acordo com o nosso entendimento de desagregação, mais adiante caracterizado. Outra expressão alemã relacionada com salto é "Sprunghaf", traduzível tanto por incoerente quanto por saltitante. Lembrar a frase com saltos repentinos na associação das idéias, destacada na caracterização do distúrbio fundamental do curso do pensamento das esquizofrenias. Esta última seria, infelizmente, um termo ambíguo. Teria Bleuler empregado ou até mesmo cunhado um neologismo, Inkohärenz, supostamente uma palavra não dicionarizada na época? A verificação em dicionários do início do século XX derrubou esta hipótese: o vocábulo Inkohärenz

1. À página 16: “... man spricht dann von 'Dissoziativem Denken' (Ziehen, 842) und 'Inkohärenz' und muß das äußere Krankheitsbild als, 'Verwirrtheit' bezeichnen” (Bleuler, 1911). Grifamos und, $e$, por ter sido traduzido, erradamente, por ou, na edição espanhola.

2. Ver nota de agradecimentos, ao final do trabalho. 


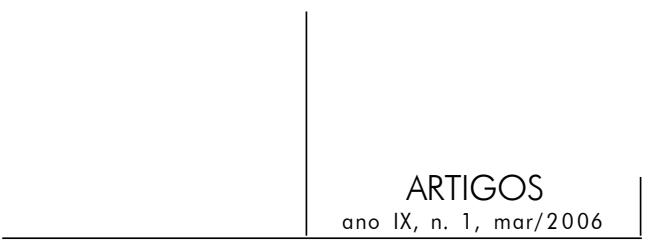

já existia no século XIX. ${ }^{3}$ Conseqüentemente, a conceituação do próprio autor, com a escolha de palavra de origem latina acrescida das aspas para ênfase, sugere que estamos diante de um tipo particular, específico, de incoerência.

Autores subseqüentes, bem como anteriores à versão inglesa da obra de Bleuler, ao consultarem a edição alemã, adotaram a expressão "incoherence" sem a ressalva de Bleuler e do seu tradutor para a língua inglesa, dando a esta expressão, traduzível por incoerência, um sentido de sinonímia com "pensamento dissociado" [desagregação do pensamento] que parece não existir no original alemão, nem mesmo na versão inglesa já referida. Noyes, em 1939, tendo como fonte de consulta a edição alemã de 1911, assim caracterizou o sintoma incoherence: "Sometimes the progression of thinking is so disorderly that one idea runs into another without logical consecution, and speech is not bound by any law. The result is a disorganization of syntactical structure with a lapse into disjointed phrases or even into parts of sentences and known as incoherence" [sem aspas]. Finalizando, escreve Noyes (1939, p. 104 e 441-3): "As might be expected incoherence occurs particularly in schizophrenia [grifado nesta transcrição], a disease in which the thinking is characteristically dominated by complexes. Much of the thinking of the schizophrenic is described as 'scattered' [disperso], which simply means that the thinking is a little less disorderly [desordenado, desregrado, transtornado] than in incoherence” (p. 104). Fica evidente, nesta frase, que Noyes está se referindo a um tipo particular ou específico de incoerência, que corresponde à "Inkohärenz" de Bleuler e à desagregação do curso do pensamento na língua portuguesa.

No capítulo sobre a esquizofrenia, Noyes escreve: "In connection with disturbances of associations mention should be made of the fact that in early schizophrenia a fairly typical flight of ideas may occur, which later tends to develop into incoherence”. Um pouco antes, o autor caracterizou o curso das associações das idéias na esquizofrenia como sendo “... shortened, fragmented, and otherwise so disturbed as to appear illogical (...) One of the earliest symptoms observed by the psychiatrist may be a certain inconsequence and odd sequence of ideas (...) As the disease becomes established there is a tendency for associative connections to become disturbed, at times becoming so broken or incomplete that the patient's utterances become fragmentary, disconnected, illogical or even unintelligible” (p. 441). A sétima edição do Kolb/Noyes (Kolb, 1971) não alterou significativamente a definição e o conceito de "incoerência". Concluindo a revisão destes livros-textos, das décadas de 1940 a 1970,

3. A palavra Inkohärenz aparece em Meyer konversations lexicon, p. 292, 1876/1887. Neulat. Museu Histórico São Leopoldo. Consulta em 13.1.04, pelo seu diretor, prof. Telmo Müller. 


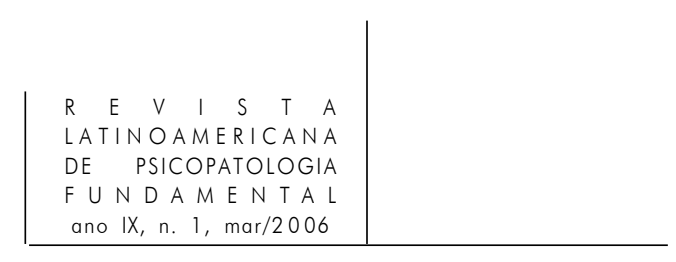

acrescente-se que, em 1950, Bleuler, Demencia Precoce já estava disponível em inglês, com "incoherence", ressalvada pelas aspas.

Estamos apenas diante de um problema de tradução e de tradição? Não, no nosso entendimento. O que dizem os pesquisadores contemporâneos, com destaque no campo da esquizofrenia, em particular nas alterações da forma e curso do pensamento?

\section{Pesquisas recentes em psicopatologia}

Em 1978, sem abordar esta confusão ou ambigüidade específica do curso do pensamento, Leme-Lopes (1978), apoiando-se em HC Rumke (1958), já havia assinalado o terreno movediço, contraditório e de incertezas da esquizofrenia (p. 3-8). Pode-se dizer ambíguo.

Em 1998, o American Journal of Psychiatry publicou um conjunto de pesquisas no campo da esquizofrenia. Ao apresentá-los, Nancy Andreasen fez importantes críticas às insuficiências dos DSMs, em particular para o diagnóstico das esquizofrenias (Andreasen, 1998, p. 1657-9). Para ela, o DSM serve apenas como um diagnostic gatekeeper, com descrições da maior parte dos transtornos intencionalmente escassas ou pobres, simples e incompletas, especialmente no caso da esquizofrenia. Declara, talvez para a surpresa de muitos, que os critérios diagnósticos dos DSMs não foram desenvolvidos para pesquisa, com os seus arquitetos bem conscientes de que suas descrições e critérios eram apenas produto de consenso [!] e que o objetivo era criar definições confiáveis, com critérios "amigáveis" para uso clínico. A editorialista pergunta: Quais são os sintomas definidores da esquizofrenia? Seriam os sintomas psicóticos que o DSM enfatiza ou os sintomas fundamentais de Bleuler, negativos e cognitivos? Bleuler, mesmo propondo uma etiologia biológica para os seus sintomas fundamentais, talvez não tenha sido perdoado pelos autodenominados neokraepelinianos, por ter tentado um entendimento psicanalítico das esquizofrenias. Os excessivamente empiristas organizadores dos DSMs excluíram os clássicos quatro "As" de Bleuler de seus critérios diagnósticos: rigidez afetiva, distúrbio da associação das idéias, autismo e ambivalência. ${ }^{4}$

4. A epistemologia e psiquiatria norte-americanas são caracterizadas por um forte empirismo (Faust). Não se deve confundir o método empirista, essencial na semiologia, com a filosofia empirista, segundo a qual só é válido o conhecimento científico baseado na observação objetiva e na mensuração. 


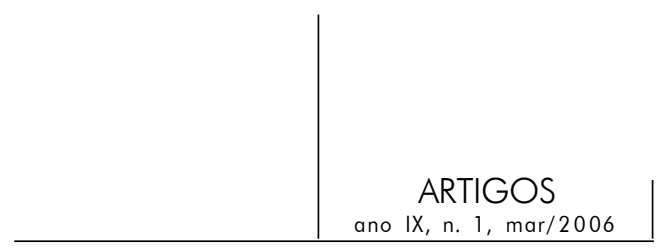

Andreasen finaliza entendendo que os pesquisadores hi-tech poderão estar dentro de dez anos defrontando-se com uma "primavera silenciosa”, 5 se a tecnologia avançar desacompanhada de clínicos prudentes e sensatos. Confirmadas as previsões de Andreasen, em 2008, cinqüenta anos depois de Rumke, o terreno da psiquiatria na esquizofrenia poderá continuar "movediço e contraditório”, pois o avanço científico, por si só, não faz desaparecer as ambigüidades (Osório; Eizirik e Goldim, 2004). Pensamos ser indispensável uma atenção às confusões e ambigüidades das definições e conceitos das principais manifestações psicopatológicas da esquizofrenia.

Um outro pesquisador contemporâneo, Weinberger (1987), para quem a esquizofrenia seria um transtorno neurodesenvolvimental, com heterogeneidade clínica e biológica (p. 660-9), tem outro entendimento dos distúrbios do curso do pensamento. Enquanto Andreasen junta fuga de idéias com derailment e loosening of associations [desagregação], Weinberger não o faz. Andreasen, mesmo com suas dúvidas, afasta-se de Bleuler. Weinberger deste se aproxima quando relaciona os prejuízos semânticos na esquizofrenia com o conceito de associações frouxas de Bleuler, ambos relativamente específicos. Para ela, associações frouxas também estariam presentes na depressão e na mania. Andreasen entende ser difícil a distinção entre a incoerência no sentido de desagregação e a incoerência que resulta das afasias de Wernicke e de Broca. Weinberger pensa diferente, inclusive a partir do mapeamento da ativação cerebral por testes de fluência semântica.

Para nós, a desagregação do curso do pensamento corresponde ao derailment dos norte-americanos ou ao knight's move dos ingleses. É o afrouxamento das associações [loosening of associations], onde a coerência está perdida não somente no conjunto, o que pode ocorrer na incoerência inespecífica ou genérica, presente em vários quadros psiquiátricos e até mesmo na normalidade, por falta de domínio de um tema, no entendimento e/ou expressão. A “incoerência”, específica, compromete também os termos e idéias deste conjunto, com perda da sintaxe, da estrutura da idéia e com o que chamaremos aqui de "frouxidão semântica", a partir das pesquisas e conceitos do grupo de Weinberger (Goldberg; Aloia; Gourovitch; Missar; Pickar; Weinberger, 1998, p. 1671-84). O pensamento de conteúdo delirante pode ser incoerente, mas não necessariamente desagregado, embora ambos - a desagregação e o delírio possam coexistir na esquizofrenia. Pacientes com transtorno delirante paranóide falam coerentemente, quanto ao curso do pensamento, mas sem desagregação. Estas diferenças não são compartilhadas por Nancy Andreasen; mas Akiskal

5. Nancy Andreasen faz alusão ao livro de Rachel Carson, Silent Spring, 1962, que expressou as preocupações com os pesticidas e inseticidas (DDT, barato e fácil de fazer) e o meio ambiente. 


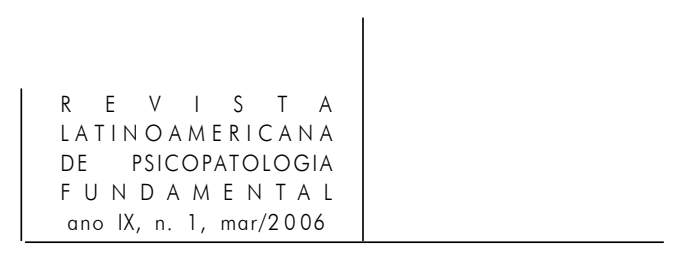

(1986) e Weinberger (1987, p. 660-9); e seu grupo de pesquisa (Goldberg; Aloia; Gourovitch; Missar; Pickar; Weinberger, 1998, p. 1671-84) destacam-nas.

Há quem proponha, como Ovsiew (2000), um epílogo para a nosologia kraepeliniana, com a sua nítida separação entre as psicoses esquizofrênicas e afetivas, estas correspondendo à enfermidade ou psicose maníaco-depressiva (p. 297-8). Kraepelin, aliás, já havia sido criticado no passado por separar a "demência precoce" das outras psicoses, no que foi defendido pelo próprio Bleuler. Segundo Ovsiew seria praticamente impossível a discriminação entre as duas já chamadas "psicoses funcionais", tanto a partir de sintomas quanto de dados biológicos. Para este autor, entre outros, teria pouco valor, portanto, a caracterização por Bleuler, no passado, e por Weinberger, na atualidade, das alterações da forma e curso do pensamento, mais sugestivas dos transtornos esquizofrênicos.

Esta pretendida superposição entre esquizofrenia e a antiga "PMD" parece ser um subproduto das confusões conceituais introduzidas no ensino e na pesquisa nos últimos cinqüenta anos pela epistemologia empirista e orientação "minimalista" nítida em parte da psiquiatria, especialmente nos Estados Unidos (Osório; Eizirik; Goldim, 2004). Uma delas é esta, entre desagregação, a "incoerência” de Bleuler, mais específica, e incoerência, termo mais abrangente. A outra é a proposta de apagar a diferença entre humor e afetividade, examinada em outras comunicações (Osório, 2000; Osório; Eizirik; Goldim; Albrecht, 2003). Em três biografias, de Vincent Van Gogh, de Kay R. Jamison e de John F. Nash, é possível verificar, claramente, a "frouxidão semântica" no caso da esquizofrenia, de Nash, e a “unidade semântica” nos casos de "PMD” (Osório, 1999; Osório, 1999a; Oliveira, 1978, p. 248; Jamison, 1996).

Young, Tanner e Meltzer examinaram o problema da baixa concordância entre diferentes sistemas diagnósticos e das definições operacionais para a esquizofrenia (Nasar, 2002). Entendem que sistemas diagnósticos distintos podem representar diferentes conceitos, daí a discrepância. Mas esta discrepância poderia também resultar de diferentes acurácias ou "eficiências" dos sistemas diagnósticos, isto é, do índice de erros sistemáticos. A acurácia/eficiência depende não só da padronização dos métodos de medida e da otimização e automatização dos instrumentos, real ou supostamente obtida pela estrutura e organização dos DSMs, mas também do treinamento, teórico e prático, dos psiquiatras na semiologia e psicopatologia do curso do pensamento.

Em instrumentos muito utilizados nas pesquisas com antipsicóticos na esquizofrenia, também vamos encontrar as mesmas ambigüidades construídas e presentes nos livros didáticos. Por exemplo, na Brief Psychiatric Rating Scale, Anchored, isto é, com definições operacionais dos níveis de gravidade (definidas por Woerner et al, 1988) há um termo abrangente, desorganização conceitual, na 
tradução de Romano e Elkis (Young, Tanner e Meltzer, 1982, p. 443-7), que corresponderia à incompreensibilidade da fala; inclui qualquer tipo de desordem formal de pensamento [formal thought disorder], por exemplo, associações frouxas, incoerência, fuga de idéias e neologismos, mas excluindo mera circunstancialidade ou fala maníaca [pressured speech no original] mesmo que acentuada. A versão original é de Overall e Gorham, 1962. A versão semiestruturada (Rhoades e Overall, 1988) foi traduzida e adaptada no Brasil por Zuardi e cols (Romano e Elkis, 1996, p. 43-9). Nesta versão o termo desorganização conceitual é também considerado sinônimo de incoerência, compreendendo os distúrbios do processo de pensamento, desde certa imprecisão na expressão verbal até produções completamente desorganizadas.

Outra importante escala é a desenvolvida por N. Andreasen. A autora, preocupada com a má compreensão e o mau uso do termo formal thought disorder, recomenda que ele deixe de ser usado, sugerindo um novo conceito, o de "disorders of thought, language, and communication", estruturando uma escala para a sua avaliação. Nesta, a autora considera flight of ideas como um derailment que ocorre rapidamente no contexto da pressão para falar. Superpõe, portanto, os conceitos do que Bleuler considera "incoerência" no sentido estrito do termo com o de fuga de idéias, em que o conceito-chave não é o de rapidez, e sim o tipo de associação de idéias (por assonância, rimas etc.).

Conclusão

Não é obrigatório, nem mesmo possível, que exista uma identidade entre as expressões de uso comum, dos dicionários não especializados e as expressões de uso técnico-científico. Exemplos não faltam. ${ }^{6}$ Mas não é desejável que existam discrepâncias muito grandes. Por exemplo, ambigüidades inconvenientes e desnecessárias seriam construídas caso os conceitos leigos e especializados de incoerência não fossem compatíveis entre si, ou que desagregação e incoerência fossem considerados sinônimos sem razões lógicas para tal.

Infelizmente os conceitos são semelhantes no léxico comum. Em Houaiss, incoerência significa falta de coerência, falta de lógica, de ligação, de nexo entre

6. Para assinalar a diferença entre o uso científico de probabilidades em Física e o uso popular do conceito, Popper declara, citando R. Von Mises, 1919, que "seria errôneo exigir que um termo científico, adequadamente definido, tivesse de corresponder, sob todos os aspectos, a uma utilização pré-científica” [popular] (Popper, 1974). 


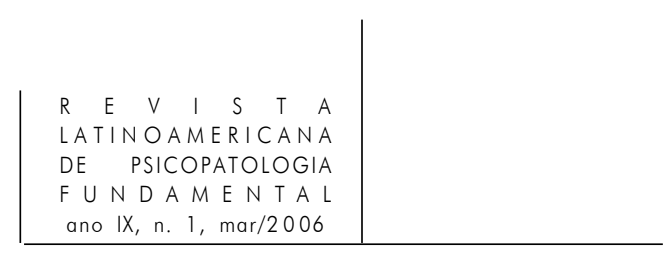

fatos, idéias, ações etc.; desarmonia, desconexão, discrepância, inconseqüência, ausência de congruência. Vem do latim cohaerentia, "conexão, coesão". Desagregação, por sua vez, significa disgregação, decomposição de um corpo em suas partes constitutivas, perda da unidade, da coesão de um conjunto organizado; dissolução, desorganização. Na psiquiatria, o mesmo que dissociação (Houaiss e Villar, 2001). No Webster's, incoherence significa lack of cohesion or adherence [falta de coesão ou adesão/aderência, conjunção], lack of continuity or relevance [falta de continuidade ou relevância]: incongruity, inconsistency [incongruência, inconsistência]. Derailment, do francês dérraillement: act or instance of derailing or being derailed Disaggregation, the separation of an aggregate into its components parts; dissociation (Webster's, 1986).

Em nome da diminuição das confusões conceituais e ambigüidades construídas no campo da psiquiatria, seria preferível usar a expressão incoerência não mais como sinônima de desagregação, recuperando-se o sentido original que Bleuler deu às características deste sintoma do curso do pensamento da esquizofrenia. Considerando, além disso, os trabalhos do grupo de Weinberger, a expressão frouxidão semântica poderia vir a ser considerada sinônima de desagregação.

\section{Agradecimentos}

A edição do Bleuler em alemão, procurada no Brasil, somente foi localizada na Biblioteca da Universidade de Toronto, por gentileza de Cintia Padoin, residente de psiquiatria da UFRGS-HCPA, em estágio naquele local, no período de julho a setembro, 2003. Agradecemos também a Luís Guilherme Streb, psiquiatra de Porto Alegre e a Mário Eduardo Costa Pereira, psiquiatra e psicanalista de Campinas, SP, pelas críticas, sugestões e esclarecimentos na língua alemã.

Ao professor Tuiskon Dick, por sua gentileza e importantes observações sobre a língua alemã.

\section{Referências}

Акiskal, H. The diagnosis in psychiatry and the mental status examination. In: Winokur, G; Clayton, P. The Medical Basis of Psychiatry. Philadelphia: Saunders, 1986 (tradução disponível no Departamento de Psiquiatria e Medicina Legal, UFRGS).

Aloia, M. S.; Gourovitch, M. L.; Missar, D.; Pickar, D.; Weinberger, D. R.; GoldBerg, T. E. Cognitive substrates of thought disorder. II. Specifying a candidate cognitive mechanism. American Journal of Psychiatry, v. 155, n. 12, p. 1677-84, December/1998. 


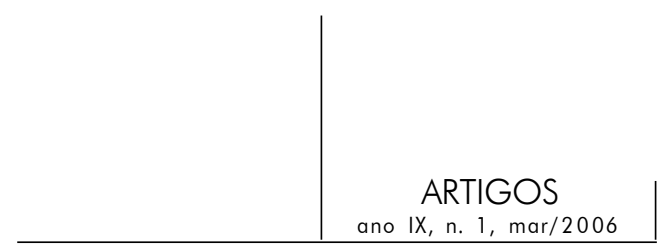

Alves Brasil, M. A.; Alves Brasil, H. Exame clínico [psiquiátrico]. In: Porto, C. C. Semiologia médica. Rio de Janeiro: Guanabara Koogan, 1990. cap. 173, p. 1089-90.

Andreasen, N. C. Scale for the assessment of thought, language, and communication. (TLC). Schizophrenia Bulletin, v. 12, n. 3, p. 473-82, 1986.

Understanding schizophrenia: a silent spring? American Journal of Psychiatry, v. 155, n. 12, p. 1657-9, December/1998 (Editorial).

Bates, B. Propedêutica médica. 4. ed. Rio de Janeiro: Guanabara Koogan, 1990 [1987, 1974-1983]. p. 85-105 [p. 90].

A Guide to Physical Examination. 3. ed. Philadelphia: Lippincott, 1983.

Ben-Dov, Y. (1995). Convite à física. Rio de Janeiro: Jorge Zahar, 1996. p. 140 [Bohr].

Bleuler, E. Dementia Praecox oder Grupper der Schizophrenien. In: Aschaffenburg, G. Handbuch der Psychiatrie. Leipzig und Wein: Franz Deuticke, 1911. p.16. 4. Abteilung, 1. Hälfte.

Dementia Praecox or the Group of Schizophrenias. New York: International Universities Press, 1950. p. 21 (tradução de J. Zinkin).

Demencia precoz; el grupo de las esquizofrenias. Buenos Aires: Paidós, 1960, p. 21-47.

Dupuy, J. P. (1994). Nas origens das ciências cognitivas. São Paulo: Unesp, 1996. p. 126-7.

FonAGY, P. (ed.). Uma revisão de portas abertas: revisão panorâmica de resultados em psicanálise. Porto Alegre: SPPA, 2001. Internet: An open door review of outcome studies in psychoanalysis. 2. ed revisited. 2002 Feb. Available from: URL: http://www.ipa.org.uk. Psychoanalysis today. http://www.wpanet.org/sectorial/docs/journalwpa5.zip.

Freedman, A; Kaplan, H. I.; SAdOCK, B. J. (eds.). Comprehensive textbook of psychiatry. 1. ed. Baltimore: Williams \& Wilkins, 1967. p. 549-50.

Gerrity, M. S.; DeVellis, R. F.; Earp, J. A. Physicians' reactions to uncertainty impatient care; a new measure and new insights. Medical Care, v. 28, n. 8, p. 724-36, 1990.

Goldberg, T. E.; Aloia, M. S.; Gourovitch, M. L.; Missar, D.; Pickard, D.; Weinberger, D. R.; Cognitive substrates of thought disorder. I. The semantic system. American Journal of Psychiatry, v. 155, n. 12, p. 1671-76, December/1998.

Houaiss, A.; Villar, M. S. Dicionário Houaiss da língua portuguesa. Rio de Janeiro: Objetiva, 2001.

JAmison, K. R. Uma mente inquieta: memórias de loucura e instabilidade de humor. São Paulo: Martins Fontes, 1996.

Kant, I. (1781). Crítica da razão pura. 2. ed. Lisboa: Calouste-Gulbenkian, 1989. Introdução, p. 36-57. 


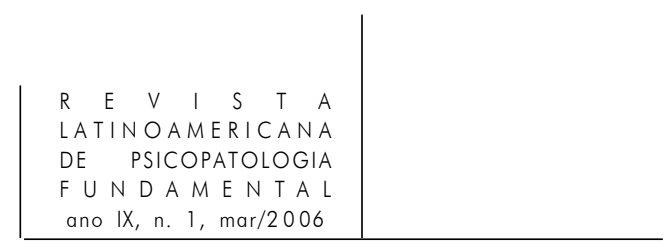

Kaplan, H. I.; SADOCK, B. J. Typical signs and symptoms of psychiatric illness In: KAplan, H. I.; Freedman, A.; SAdock, B. J. (eds.). Comprehensive textbook of psychiatry. 3. ed. Baltimore: Williams \& Wilkins, 1980. chap. 12.4, p. 925.

Typical signs and symptoms of psychiatric illness In: Kaplan, H. I.; FreEdMAN, A.; SADOCK, B. J. (eds.). Comprehensive textbook of psychiatry. 4. ed. Baltimore: Williams \& Wilkins, 1985. chap. 12.4, p. 500.

Typical signs and symptoms of psychiatric illness. In: KaPLAN, H. I.; FrEEDMAN, A.; SADOCK, B. J. (eds.). Comprehensive textbook of psychiatry. 5. ed. Baltimore: Williams \& Wilkins, 1990. p. 472.

Typical signs and symptoms of psychiatric illness. In: Kaplan, H. I.; Freedman, A.; SADock, B. J. (eds.). Comprehensive textbook of psychiatry. 6. ed. Baltimore: Williams \& Wilkins, 1995. p. 540.

Typical signs and symptoms of psychiatric illness. In: Kaplan, H. I.; Freedman, A.; SADоcK, B. J. (eds.). Comprehensive textbook of psychiatry. 7. ed. Baltimore: Lippincott Williams \& Wilkins, 2000. p. 687.

KolB, L. C. Noyes Psiquiatria clínica moderna. 4. ed. México: La Prensa Médica Mexicana, 1971(1968, 7. ed. nos Estados Unidos) (Arthur P. Noyes faleceu em 1963).

Koyré, A. (1973). Traduttore-traditore. A propósito de Copérnico e de Galileu. In: Estudos de história do pensamento científico. Rio de Janeiro: Forense Universitária, 1982 [extraído de Isís, 1943, 34(95), p. 209-10].

Lang da SiLveIra, F. Comunicação pessoal, 2003.

Langenscheidt's. Taschenwörterbuch der Portugiesischen und Deutschen Sprache. Berlin und München: Langscheidt, 1968, p. 336 (incoerência, Hanglosigkeit, Sprunghaftsigkeit) e 1064 (Sprunghaftt: saltitante, incoerente).

Leme-Lopes, J. L. Conceito de esquizofrenia: um "s" a menos. Jornal Brasileiro de Psiquiatria, v. 27, n. 1-4, p. 3-8, 1978.

LinN, L. Clinical manifestations of psychiatric disorders. In: Kaplan, H. I.; FreEdMan, A.; SADock, B. J. (eds). Comprehensive textbook of psychiatry. 2. ed. Baltimore: Williams \& Wilkins, 1975. chap. 13.1

LORD, B. Uses of quotation marks for emphasis. Consulta Internet. http://alt-usageenglish.org/quotes_for emphasis. html, consulta em 10/9/03.

Manley, M. R. S. Psychiatric interview, history, and mental status examination. In: SADock, B. J. \& SADock, V. A. (eds.). Kaplan \& Sadock's Comprehensive Textbook of Psychiatry. 7. ed. Philadelphia: Lippincott, 2000. chap 7.1, p. 652-65.

McHugh, P. \& Slavney, P. As perspectivas da psiquiatria. Porto Alegre: Artes Médicas, 1989. 


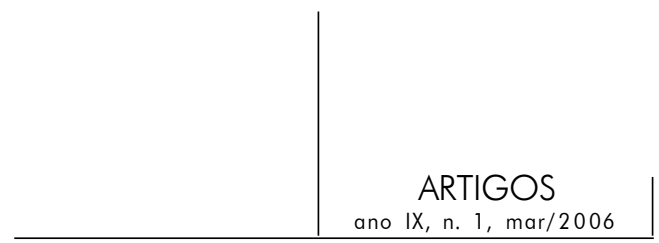

Medical Research Council. Institute of Psychiatry. In: Glossário das definições dos sintomas incluídos na 9ae ed. do PSE. São Paulo: USP-HC, 1983 (tradução para o português de Raul Caetano, Valentim Gentil Filho e colaboradores).

MerriLl, J. M. et al. Uncertainties and ambiguities; measuring how medical students cope. Medical Education, v. 28, p. 316-22, 1994.

Meyer. Konversations lexicon, p. 292, 1876/1887. Neulat. Museu Histórico São Leopoldo. Consulta feita em 13.1.04, pelo seu próprio diretor, prof. Telmo Müller. [Zusammenhanglosig-keit.] [Enciclopédia]

NASAR, S. Uma mente brilhante (origem do filme sobre John F. Nash). Rio de Janeiro: Record, 2002.

Noyes, A. P. Modern clinical psychiatry. Philadelphia: Saunders, 1939. p. 104 e 441-3.

Oliveira, F. de. Van Gogh. In: Literatura e civilização. Rio de Janeiro: Difel, Brasília: Instituto Nacional do Livro, MEC, 1978. p. 248.

OsóRIo, C. M. S. Depressão bipolar: Van Gogh, 1999 (manuscrito não publicado, revisado em 2003). p. 8, 1999.

O carteiro e o pintor. Jornal do Centro de Estudos Luís Guedes, v. 10, n. 40,

O diagnóstico nosográfico [CID] e o diagnóstico psicanalítico na psiquiatria: convergentes, divergentes, complementares? Quando, como e por que? Participação em mesa-redonda. XX Jornada Sul-Rio-Grandense de Psiquiatria Dinâmica. Gramado, outubro 2000 [manuscrito].

Osório, C. M. S.; Eizirik, C. L.; Goldim, J. R.; Albrecht, R. Afetos, emoções, sentimentos, temperamento e humor; uma proposta de exame do estado mental com mais clareza conceitual, menos confusão e menos ambigüidades. Porto Alegre: UFRGS, Faculdade de Medicina, PPG Ciências Médicas: Psiquiatria. Dissertação de mestrado. Trabalho utilizado para o EGQ, Exame Geral de Qualificação. Novembro, 2003.

OsóRIO, C. M. S; EIZIRIK, C. L.; GoldIM, J. R. Incerteza, ambigüidade e formação médica; uma revisão da literatura. 2004. Dissertação (mestrado em Psiquiatria), Porto Alegre: UFRGS, Faculdade de Medicina, PPG Ciências Médicas: Psiquiatria.

Ovsiew, F. The end to Kraepelinian nosology? Journal of Neuropsychiatry and Clinical Neurosciences, v. 12, n. 3, p. 297-8, summer 2000 (Editorial).

Popper, K. R. (1934) A lógica da pesquisa científica. 2. ed. São Paulo: Cultrix, 1974. p. 168.

Porto, C. C. Semiologia médica. Rio de Janeiro: Guanabara-Koogan, 1990. p. 1089-90 [Exame psiquiátrico, Parte 14].

REID, Th. A. Essays on the intellectual power of Man (1785). Columbia World of Quotations 1966 Number 46182. Internet, março, 2003 [explication of words]. 


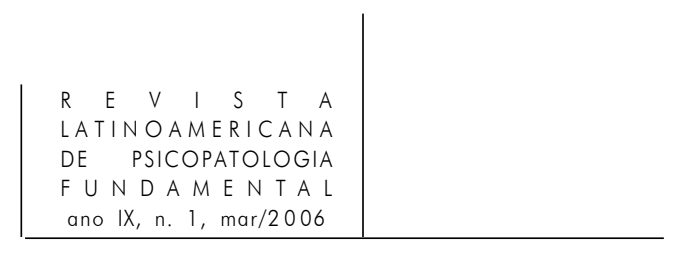

Romano, F.; Elkis, H. Tradução e adaptação de um instrumento de avaliação psicopatológica das psicoses: a escala breve de avaliação psiquiátrica - versão ancorada (BPRS-A). Jornal Brasileiro de Psiquiatria, v. 45, p. 43-9, 1996.

RónAI, P. (1975). A tradução vivida. 2. ed. rev. Rio de Janeiro: Nova Fronteira, 1991. p. 62.

Schor, R.; Pilpel, D.; Benbassat, J. Tolerance of uncertainty of medical students and practicing physicians. Medical Care, v. 38, n. 3, p. 272-80, March/2000 (http:// gateway1.ovid.com/ovidweb.cgi, 10/5/02).

WeBster's. Third new international dictionary of the English language unabridged. Springfield, Mass.: Merriam Webster, 1986.

WEINBERGER, D. R. Implications of normal brain development for the pathogenesis of schizophrenia. Archives of General Psychiatry, v. 44, p. 660-9, July/1987.

Young, M. A.; Tanner, M. A.; Meltzer, H. Y. Operational definitions of schizophrenia. What do they identify? Journal of Nervous and Mental Diseases, v. 170, n. 8, p. 4437, August/1982.

Zuardi, A.W.; Loureiro, S. R.; Rodrigues, C. R. C. et al. Estudo da estrutura fatorial, fidedignidade e validade da tradução e adaptação para o português da Escala de Avaliação Psiquiátrica Breve (BPRS) modificada. Revista da ABP-APAL, v. 16, p. 63-8, 1994.

\section{Resumos}

En la investigación sobre tolerancia, reacciones a la ambigüedad y a la incertidumbre en la medicina, fueron identificadas ambigüedades y confusiones conceptuales que dificultan el proceso de enseñanza-aprendizaje y la investigación. Son ejemplos los conceptos ambiguos y/o problemas en la traducción de términos psicopatológicos. Disgregación e incoherencia en el curso del pensamiento son frecuentemente definidos y conceptualizados prácticamente como sinónimos, muy probablemente a partir de una distorsión en la comprensión de "Inkohärenz", expresión original de Bleuler. Los avances en la investigación psicopatológica, en la enseñanza y en la práctica clínica dependen de la claridad conceptual, y de la precisión en la nomenclatura, las definiciones y en la descripción de los síntomas psicopatológicos. Se revisaron los conceptos y definiciones dados a las alteraciones del curso y la forma del pensamiento en todas las ediciones de Kaplan y Sadock, juntamente con otros textos didácticos. Se cotejaron también los puntos de vista de Andreasen y Weinberger en relación a ese tema de semiología psiquiátrica.

Palabras claves: Psicopatología, investigación (curso y forma del pensamiento), incoherencia, disgregación 


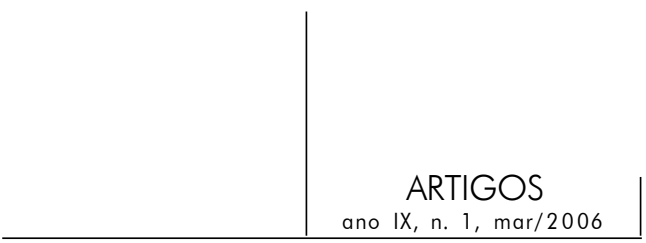

L'investigation en ce qui concerne la tolérance et les réactions à l'ambiguïté et à l'incertitude de la Médecine, a rencontré quelques obstacles pour l'apprentissage et la recherche en Psychopathologie. Parmis les dernières on trouve des concepts confus et des problèmes de traduction des expressions de Psychopathologie. Dérraillement et incohérence sont presque définies comme synonymes tenant en compte une probable distorsion au niveau de la compréhension de "Inkohärenz", l'expression original de Bleuler. Les progrès de la recherche, de l'enseignement et de la pratique clinique en Psychopathologie sont dépendants de la clarté conceptuel et de la définition des symptômes en Psychopathologie descriptive. Les concepts et les définitions des altérations de l'évolution et de la forme de la pensée dans toutes les éditions de Kaplan et Sadock's aussi que dans d'autres textes didactiques sont revus. Les avis de Andreasen et Weinberger a propos du sujet Sémiologie Psychiatrique sont comparés.

Mots clés: Psychopathologie, recherche, incohérence, dérraillement

In the research on and reactions towards ambiguity and uncertainty in medicine, certain types of obstacles have been identified in the learning-teaching process and in research in psychopathology. Examples include the confusing concepts and problems related to mis-translated psychopathological terms. The symptoms of desegregation and inconsistency during thought have been defined and conceptualized virtually as synonyms, due to a distortion in the understanding of Bleuler's original expression "Inkohärenz." The advances in psychopathological research, teaching and clinical practice depend on the conceptual clarity and accuracy in the nomenclature of descriptive psychopathology. The concepts and definitions of the changes in the course or form of thinking in all the editions of Kaplan and Sadock, as well as terms in other textbooks, are taken up here. Andreasen's and Weinberger's points of view are also compared in this theme in psychiatric semiology.

Key words: Psychopathology, research (course and way thinking), inconsistency, disaggregation

Versão inicial recebida em outubro de 2005

Aprovado para publicação em janeiro de 2006 


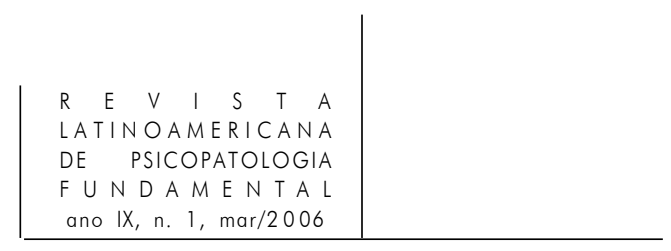

\section{Apêndice 1}

Palavras de Vincent a Gauguin, em uma das tumultuosas conversas em Arles, circa 1888? O quadro Red Vineyard já estava pronto. O texto exemplifica a unidade semântica, ou seja, a ausência de desagregação ou afrouxamento de associações. Talvez seja um belo exemplo de um discurso hipomaníaco ou maníaco [dependendo do afeto, exaltação em que o mesmo seja pronunciado] com, no máximo, fuga de idéias, pela riqueza das associações e fluidez.

Quando pinto o sol, quero que todo o mundo o sinta rodando no espaço a uma velocidade tremenda, desferindo ondas de luz e calor. Quando pinto um trigal, quero que todo o mundo sinta a luta dos átomos dentro das espigas até o triunfo do amadurecimento. Quando pinto uma maçã, quero fazer sentir o suco da fruta, por baixo da casca, e as sementes, no miolo, ansiando pela germinação. Olhe esta vinha. Não parece que os cachos de uvas vão rebentar, de tão maduras? Olhe para esta encosta: todos sentem que milhares de toneladas de água já correram pelos flancos escarpados. Quando pinto o retrato de um homem, procuro captar-lhe a vida toda, tudo o que viu, fez e sofreu. Os campos que alimentam o trigo, a água que desce a encosta, o suco da uva e o passado de uma criatura humana - tudo é a mesma coisa. A única unidade da vida é a unidade do ritmo. Tudo dança dentro desse ritmo universal: homens, maçãs, encostas, campos lavrados, carros de trigo, casas, cavalos e o próprio sol. A substância que hoje pulsa dentro de você e a maçã são uma mesma coisa. Quando pinto um camponês trabalhando no campo, quero exprimir que ele tem raízes no solo, como o trigo, e que a força da terra o anima. Quero que se sinta o sol entrando, entrando no homem, no campo, no trigo, no arado e nos cavalos, $e$ recebendo a força que dele emana. Quando você começar a sentir o ritmo universal dentro do qual tudo se move, então você estará começando a compreender a vida. E Deus é isso!

Fonte: Oliveira, Franklin de [1913-?]. Literatura e civilização. Rio de Janeiro: MECDifel, 1978

\section{Apêndice 2}

Glossário dos sintomas do curso/forma do pensamento, nas sete edições do atual Kaplan \& Sadock, resumo.

- $1^{\text {a }}$ ed.: sem glossário; com separação entre distúrbios do curso do pensamento e distúrbios da forma, dereísmo e autismo; word salad = fala com neologismos e frases ou palavras incoerentes, sem significado lógico, ouvidas em formas graves de esquizofrenia; sem loosening of associations ou derailment; incoherence definida como resultado da desordem do pensamento: pensamentos sem seqüência 


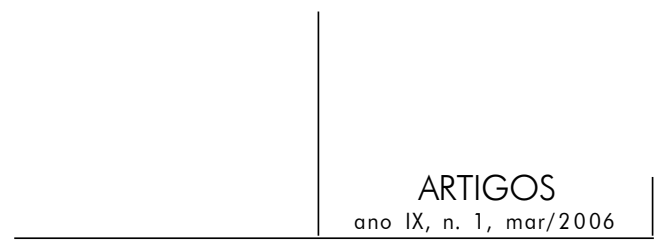

lógica, incompreensíveis; forma mais leve = scattering, dispersão?; tangenciality, relacionada com circunstancialidade.

- 2a ed.: c/glossário; alterações da forma e do curso vistas conjuntamente; incoherence = distúrbio da estrutura das associações, running together de pensamentos sem conexão lógica, com desorganização; blocking = interruption... unconscious origin; loosening of associations = fluxo de pensamento casual, sem propósito, ilógico, confuso, incorreto, abrupto e bizarro; sintoma fundamental da esquizofrenia, para Bleuler; derailment = não tem; word salad, distúrbio da estrutura das associações = mistura incoerente de palavras ou frases, comumente vista em estágios avançados da esquizofrenia; tangenciality = diferenciada da circunstancialidade; naquela o objetivo nunca está claramente definido ou nunca é alcançado; o diálogo sai fora dos trilhos.

- $\quad 3^{\mathrm{a}}$ ed.: com glossário; incoherence = running together of thoughts..., como na $2^{\mathrm{a}}$ ed.; no glossário, incoherence é definida como comunicação inconexa, ilógica e incompreensível; loosening of associations, derailment = não aparecem, embora exista blocking = interruption... of unconscious origin; word salad $=$ igual à $2^{\text {a }}$ ed., com remessa à incoerência; no capítulo de neurologia, fala-se de word salad como jargonofasia, embora não usando este termo, mas diferenciando do discurso esquizofrênico.

- $4^{\mathrm{a}}$ ed.: incoherence = running together of thoughts, sem conexão lógica, resultando em desorganização; derailment = gradual ou súbito desvio, sem bloqueio, na seqüência do pensamento, este sendo vago e não focalizado, como é o loosening of associations = definido como fluxo de pensamento, vago, não focalizado e ilógico; sint. fundamental da esquizofrenia, para Bleuler, passível de decodificação psicodinâmica; word salad = mistura de palavras ou frases sem significado lógico; blocking = igual à $2^{\mathrm{a}}$ e $3^{\mathrm{a}}$ eds; tangenciality = similar à $2^{\mathrm{a}}$ ed.

- $\quad 5^{\mathrm{a}}$ ed.: formal thought disorder = loosened associations + neologisms + illogical constructs. Incoherence = running together... de pensamentos ou palavras sem conexão lógica, desorganização incomprensível. Derailment = desvio gradual ou súbito na seqüência de pensamento, às vezes sinônimo de afrouxamento de associações. Este, loosening of associations é o fluxo de pensamento com idéias mudando de um assunto para outro sem relação alguma; se grave, o discurso tornase incoerente; podendo haver uma lógica privada; manifestação central da esquizofrenia, cf. Bleuler. Às vezes o afrouxamento das associações que ocorre na esquizofrenia é chamado de derailment. Word salad é a mistura incoerente de palavras ou frases, resultando da intensificação do loosening of associations até perto da incoerência. Blocking é a interrupção abrupta da seqüência de pensamentos, sem concluí-los.

- 6 ed.: incoherence = pensamento geralmente não compreensível, running together o thoughts, sem conexões lógicas ou gramaticais, resultando em desorganização; 


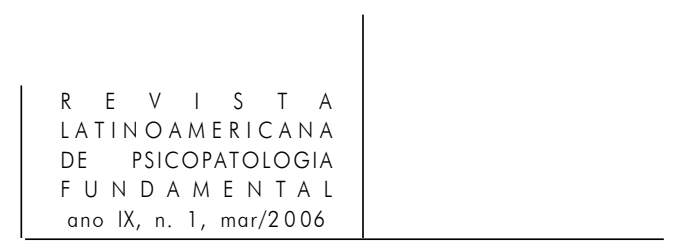

perseveração, intrusões e incoerência seriam singulares transtornos da forma de pensamento em condições neuropsiquiátricas; loosening of associations = como na $5^{\text {a }}$ ed.; para alguns teóricos, o aspecto fundamental na esquizofrenia; derailment = definido como na $5^{\mathrm{a}}$ ed.; word salad = como na $5^{\mathrm{a}}$ ed., completa desordem da fala; blocking = interrupção abrupta da seqüência do pensamento, antes que este tenha sido terminado; tangenciality = incapacidade de associações de pensamento dirigidas a um objetivo: o paciente nunca chega ao ponto ou objetivo desejado; Vorbereiden seria uma forma de tangencialidade; esta, por sua vez, seria uma forma leve de derailment; formal thought disorder = distúrbio da forma, não do conteúdo = como na $5^{\mathrm{a}}$ ed.

- $7^{\mathrm{a}}$ ed.: formal thought disorder $=$ como na $5^{\mathrm{a}}$ e na $6^{\mathrm{a}}$ eds; incoherence $=$ comunicação desconexa, sem associação lógica, desorganizada, incompreensível, com remessa à word salad = mistura de palavras e frases incoerentes, essencialmente incompreensível, comumente vista em casos bem avançados de esquizofrenia; seria mais comum no delirium e demências corticais, como Alzheimer; derailment, descarrilamento, "sair fora dos trilhos" = gradual ou súbito desvio na seqüência de pensamentos, sem bloqueio; o descarrilamento seria considerado, às vezes, como sinônimo de afrouxamento das associações = loosening of associations = distúrbio da fala ou do pensamento caracteristicamente esquizofrênico, com transtorno na progressão lógica dos pensamentos, com fracasso na comunicação verbal adequada. Transtorno específico da esquizofrenia, o mais valioso critério diagnóstico, mas requerendo bons conhecimentos de psicopatologia para não ser confundido com outras formas de distúrbio do pensamento: fuga maníaca de idéias, desintegração dos processos do pensamento por obscurecimento da consciência; raciocínio prejudicado por fadiga ou distração. Pelo teste dos provérbios dá para confundir pacientes com afrouxamento das associações com pacientes com fuga de idéias. Resultaria da perda do propósito da linguagem na esquizofrenia. Os autores remetem o leitor a tangenciality = maneira de falar oblíqua, contrária de progressiva, irrelevante, na qual a idéia central não é comunicada; forma mais leve de derailment; Vorbereiden seria uma forma de tangencialidade; blocking = visto na esquizofrenia, súbita quebra da seqüência do pensamento.

\section{Apêndice 3}

Síntese do Glossário de Psicopatologia [CMS Osório]

1. Fuga de idéias: não é simplesmente a velocidade rápida, como muitas fontes sug rem, entre elas Kaplan \& Sadock, e sim o modo como são feitas as ass ciações. Sacrif|ca-se a semelhança e/ou drelação interna em favor de 
continuidades e semelhança externa. A associação de idéias é feita por assonância ou sonoridade/clanging”, o que não deixa de ser uma associação de idéias governada por certas leis, diferentemente do que se vê na esquizofrenia.

2. Incoerência/incoherence: é uma alteração do curso do pensamento que depende de outros distúrbios, o que já a diferencia da desagregação, esta última relativamente independente. Incoerência pode resultar da própria desagregação, mas o inverso não é verdadeiro. Incoerência é conseqüência também de fuga de idéias muito intensa, de taquilalia, de verborragia, da obnubilação ou turvação da consciência (como no delirium), de parafasia e jargonofasia e da deficiência mental. Pessoas normais, em situações especiais: provas escolares ou acadêmicas, ao expor um tema, podem mostrar incoerência no curso do pensamento, quando se combinam ansiedade e desconhecimento do assunto. Uma pessoa que não domina um outro idioma pode também falar de modo incoerente ou sem conexão, mas sem nenhum significado psicopatológico.

3. Desagregação, derailment, knight's move, afrouxamento das associações: o curso não segue uma linha reta, ziguezagueia, ficando truncado, inconcluso, descontínuo, “em saltos”. O cavalo do xadrez, além do movimento “em L”, é a única peça que "salta” por cima de outras. A coerência está perdida não somente no conjunto (o que pode ocorrer na incoerência), mas também nos termos e idéias deste conjunto, com perda da sintaxe e da estrutura da idéia (conforme a brasileira Iracy Doyle e o já citado Noyes). Corresponde também ao que se pode chamar de "frouxidão semântica”, a partir das pesquisas e conceitos de Weinberger, isto é, perde-se a unidade semântica do discurso normal. Por exemplo, uma pessoa está falando de camarões e peixes e, em um salto, pula para o tema Volkswagen e lápis de cera. O pensamento de conteúdo delirante pode ser incoerente, mas não necessariamente desagregado, embora eles - desagregação e delírio - possam coexistir, na esquizofrenia. Pacientes com transtorno delirante paranóide falam coerentemente (no que se refere ao curso do pensamento) e sem desagregação. Estas diferenças não são compartilhadas por Nancy Andreasen, mas Akiskal e outros autores, como Weinberger, destacam-nas. 